\title{
Fuzzy fractional calculus revisited
}

George A. Anastassiou

Department of Mathematical Sciences, University of Memphis,Memphis, TN 38152, U.S.A.

Received: 13 June 2021, Accepted: 16 June 2021

Published online: 16 July 2021.

\begin{abstract}
Here we study very general fuzzy fractional integral-differential operators, give their basic properties and derive related Opial and Hardy type inequalities. Then we describe a list of specific such operators of singular and non-singular kernels that fulfill this general theory.
\end{abstract}

Keywords: Fuzzy Fractional Calculus, fuzzy fractional integral inequalities, singular and non-singular kernels.

\section{Fuzzy mathematical analysis needed}

This work is a continuation of author's [6], Chapter 34. We need the following basic fuzzy background.

Definition 1. (see [24]) Let $\mu: \mathbb{R} \rightarrow[0,1]$ with the following properties

(i) is normal, i.e., $\exists x_{0} \in \mathbb{R} ; \mu\left(x_{0}\right)=1$.

(ii) $\mu(\lambda x+(1-\lambda) y) \geq \min \{\mu(x), \mu(y)\}, \forall x, y \in \mathbb{R}, \forall \lambda \in[0,1]$ ( $\mu$ is called a convex fuzzy subset).

(iii) $\mu$ is upper semicontinuous on $\mathbb{R}$, i.e. $\forall x_{0} \in \mathbb{R}$ and $\forall \varepsilon>0$, $\exists$ neighborhood $V\left(x_{0}\right): \mu(x) \leq \mu\left(x_{0}\right)+\varepsilon, \forall x \in V\left(x_{0}\right)$.

(iv) The set $\overline{\sup p(\mu)}$ is compact in $\mathbb{R}$, (where $\sup p(\mu):=\{x \in \mathbb{R}: \mu(x)>0\}$ ).

We call $\mu$ a fuzzy real number. Denote the set of all $\mu$ with $\mathbb{R}_{\mathscr{F}}$.

E.g. $\chi_{\left\{x_{0}\right\}} \in \mathbb{R}_{\mathscr{F}}$, for any $x_{0} \in \mathbb{R}$, where $\chi_{\left\{x_{0}\right\}}$ is the characteristic function at $x_{0}$.

For $0<r \leq 1$ and $\mu \in \mathbb{R}_{\mathscr{F}}$ define

$$
[\mu]^{r}:=\{x \in \mathbb{R}: \mu(x) \geq r\}
$$

and

$$
[\mu]^{0}:=\overline{\{x \in \mathbb{R}: \mu(x) \geq 0\}} .
$$

Then it is well known that for each $r \in[0,1],[\mu]^{r}$ is a closed and bounded interval on $\mathbb{R}([15])$.

For $u, v \in \mathbb{R}_{\mathscr{F}}$ and $\lambda \in \mathbb{R}$, we define uniquely the sum $u \oplus v$ and the product $\lambda \odot u$ by

$$
[u \oplus v]^{r}=[u]^{r}+[v]^{r}, \quad[\lambda \odot u]^{r}=\lambda[u]^{r}, \forall r \in[0,1]
$$

where

$-[u]^{r}+[v]^{r}$ means the usual addition of two intervals (as substes of $\mathbb{R}$ ) and

$-\lambda[u]^{r}$ means the usual product between a scalar and a subset of $\mathbb{R}$ (see, e.g. [24]).

\footnotetext{
*Corresponding author e-mail: ganastss@memphis.edu
} 
Notice $1 \odot u=u$ and it holds

$$
u \oplus v=v \oplus u, \lambda \odot u=u \odot \lambda
$$

If $0 \leq r_{1} \leq r_{2} \leq 1$ then $[u]^{r_{2}} \subseteq[u]^{r_{1}}$. Actually $[u]^{r}=\left[u_{-}^{(r)}, u_{+}^{(r)}\right]$, where $u_{-}^{(r)} \leq u_{+}^{(r)}, u_{-}^{(r)}, u_{+}^{(r)} \in \mathbb{R}, \forall r \in[0,1]$. For $\lambda>0$ one has $\lambda u_{ \pm}^{(r)}=(\lambda \odot u)_{ \pm}^{(r)}$, respectively. Define

$$
D: \mathbb{R}_{\mathscr{F}} \times \mathbb{R}_{\mathscr{F}} \rightarrow \mathbb{R}_{\mathscr{F}}
$$

by

$$
D(u, v):=\sup _{r \in[0,1]} \max \left\{\left|u_{-}^{(r)}-v_{-}^{(r)}\right|,\left|u_{+}^{(r)}-v_{+}^{(r)}\right|\right\}=\sup _{r \in[0,1]} \operatorname{Hausdorff} \operatorname{distance}\left([u]^{r},[v]^{r}\right),
$$

where $[v]^{r}=\left[v_{-}^{(r)}, v_{+}^{(r)}\right] ; u, v \in \mathbb{R}_{\mathscr{F}}$. We have that $D$ is a metric on $\mathbb{R}_{\mathscr{F}}$. Then $\left(\mathbb{R}_{\mathscr{F}}, D\right)$ is a complete metric space, see [24], [25], with the properties

$$
\begin{aligned}
D(u \oplus w, v \oplus w) & =D(u, v), \forall u, v, w \in \mathbb{R}_{\mathscr{F}}, \\
D(k \odot u, k \odot v) & =|k| D(u, v), \quad \forall u, v \in \mathbb{R}_{\mathscr{F}}, \forall k \in \mathbb{R}, \\
D(u \oplus v, w \oplus e) & \leq D(u, w)+D(v, e), \quad \forall u, v, w, e \in \mathbb{R}_{\mathscr{F}} .
\end{aligned}
$$

Here $\Sigma^{*}$ stands for fuzzy summation and $\widetilde{0}:=\chi_{\{0\}} \in \mathbb{R}_{\mathscr{F}}$ is the neutral element with respect to $\oplus$, i.e.,

$$
u \oplus \widetilde{0}=\widetilde{0} \oplus u=u, \forall u \in \mathbb{R}_{\mathscr{F}} .
$$

Denote

$$
D^{*}(f, g):=\sup _{x \in[a, b]} D(f, g)
$$

where $f, g:[a, b] \rightarrow \mathbb{R}_{\mathscr{F}}$. We mention

Definition 2. Let $f:[a, b] \subseteq \mathbb{R} \rightarrow \mathbb{R}_{\mathscr{F}}$, we define the (first) fuzzy modulus of continuity of $f$ by

$$
\omega_{1}^{(\mathscr{F})}(f, \delta)=\sup _{x, y \in[a, b],|x-y| \leq \delta} D(f(x), f(y)), \delta>0 .
$$

We define by $C_{\mathscr{F}}^{U}([a, b])$ the space of fuzzy uniformly continuous functions from $[a, b] \rightarrow \mathbb{R}_{\mathscr{F}}$, also $C_{\mathscr{F}}([a, b])$ is the space of fuzzy continuous functions on $[a, b]$. It is clear that

$$
C_{\mathscr{F}}^{U}([a, b])=C_{\mathscr{F}}([a, b])
$$

We mention

Proposition 1. ([3]) Let $f \in C_{\mathscr{F}}^{U}([a, b])$. Then $\omega_{1}^{(\mathscr{F})}(f, \delta)<\infty$, for any $\delta>0$.

Proposition 2. ([3]) It holds

$$
\lim _{\delta \rightarrow 0} \omega_{1}^{(\mathscr{F})}(f, \delta)=\omega_{1}^{(\mathscr{F})}(f, 0)=0
$$

iff $f \in C_{\mathscr{F}}^{U}([a, b])$.

Proposition 3. ([3]) Here $[f]^{r}=\left[f_{-}^{(r)}, f_{+}^{(r)}\right], r \in[0,1]$. If $f \in C_{\mathscr{F}}([a, b])$ then $f_{ \pm}^{(r)} \in C([a, b])$, for $r \in[0,1]$, in fact these are equicontinuous families, respectively in \pm . Furthermore $f$ is a fuzzy bounded function.

We need 
Definition 3. Let $x, y \in \mathbb{R}_{\mathscr{F}}$. If there exists $z \in \mathbb{R}_{\mathscr{F}}: x=y \oplus z$, then we call $z$ the $H$-difference on $x$ and $y$, denoted $x-y$.

Definition 4. ([24]) Let $T:=\left[x_{0}, x_{0}+\beta\right] \subset \mathbb{R}$, with $\beta>0$. A function $f: T \rightarrow \mathbb{R}_{\mathscr{F}}$ is $H$-differentiable at $x \in T$ if there exists an $f^{\prime}(x) \in \mathbb{R}_{\mathscr{F}}$ such that the limits (with respect to $D$ )

$$
\lim _{h \rightarrow 0+} \frac{f(x+h)-f(x)}{h}, \lim _{h \rightarrow 0+} \frac{f(x)-f(x-h)}{h}
$$

exist and are equal to $f^{\prime}(x)$. We call $f^{\prime}$ the $H$-derivative or fuzzy derivative of $f$ at $x$.

Above is assumed that the $H$-differences $f(x+h)-f(x), f(x)-f(x-h)$ exist in $\mathbb{R}_{\mathscr{F}}$ in a neighborhood of $x$. We denote by $C_{\mathscr{F}}^{N}([a, b]), N \geq 1$, the space of all $N$-times continuously fuzzy differentiable functions from $[a, b]$ into $\mathbb{R}_{\mathscr{F}}$. We mention

Theorem 1. ([17]) Let $f:[a, b] \rightarrow \mathbb{R}_{\mathscr{F}}$ be H-fuzzy differentiable. Let $t \in[a, b], 0 \leq r \leq 1$. Clearly

$$
[f(t)]^{r}=\left[f(t)_{-}^{(r)}, f(t)_{+}^{(r)}\right] \subseteq \mathbb{R} .
$$

Then $(f(t))_{ \pm}^{(r)}$ are differentiable and

$$
\left[f^{\prime}(t)\right]^{r}=\left[\left(f(t)_{-}^{(r)}\right)^{\prime},\left(f(t)_{+}^{(r)}\right)^{\prime}\right]
$$

I.e.

$$
\left(f^{\prime}\right)_{ \pm}^{(r)}=\left(f_{ \pm}^{(r)}\right)^{\prime}, \quad \forall r \in[0,1]
$$

Remark.([2]) Let $f \in C_{\mathscr{F}}^{N}([a, b]), N \geq 1$. Then by Theorem 1 we obtain

$$
\left[f^{(i)}(t)\right]^{r}=\left[\left(f(t)_{-}^{(r)}\right)^{(i)},\left(f(t)_{+}^{(r)}\right)^{(i)}\right]
$$

for $i=0,1,2, \ldots, N$, and in particular we have that

$$
\left(f^{(i)}\right)_{ \pm}^{(r)}=\left(f_{ \pm}^{(r)}\right)^{(i)}
$$

for any $r \in[0,1]$, all $i=0,1,2, \ldots, N$.

Note 1. ([2]) Let $f \in C_{\mathscr{F}}^{N}([a, b]), N \geq 1$. Then by Theorem 1 we have $f_{ \pm}^{(r)} \in C^{N}([a, b])$, for any $r \in[0,1]$.

We need also a particular case of the Fuzzy Henstock integral $\left(\delta(x)=\frac{\delta}{2}\right)$, see [24].

Definition 5. ([13], p. 644) Let $f:[a, b] \rightarrow \mathbb{R}_{\mathscr{F}}$. We say that $f$ is Fuzzy-Riemann integrable to $I \in \mathbb{R}_{\mathscr{F}}$ if for any $\varepsilon>0$, there exists $\delta>0$ such that for any division $P=\{[u, v] ; \xi\}$ of $[a, b]$ with the norms $\Delta(P)<\delta$, we have

$$
D\left(\sum_{P}^{*}(v-u) \odot f(\xi), I\right)<\varepsilon .
$$

We write

$$
I:=(F R) \int_{a}^{b} f(x) d x
$$

We mention.

Theorem 2. ([15]) Let $f:[a, b] \rightarrow \mathbb{R}_{\mathscr{F}}$ be fuzzy continuous. Then

$$
(F R) \int_{a}^{b} f(x) d x
$$


exists and belongs to $\mathbb{R}_{\mathscr{F}}$, furthermore it holds

$$
\left[(F R) \int_{a}^{b} f(x) d x\right]^{r}=\left[\int_{a}^{b}(f)_{-}^{(r)}(x) d x, \int_{a}^{b}(f)_{+}^{(r)}(x) d x\right]
$$

$\forall r \in[0,1]$.

Theorem 3. ([24]) Let $f \in C_{\mathscr{F}}([a, b])$ and $c \in[a, b]$. Then

$$
(F R) \int_{a}^{b} f(x) d x=(F R) \int_{a}^{c} f(x) d x+(F R) \int_{c}^{b} f(x) d x .
$$

Theorem 4. ([15]) Let $f, g \in C_{\mathscr{F}}([a, b])$ and $c_{1}, c_{2} \in \mathbb{R}$. Then

$$
(F R) \int_{a}^{b}\left(c_{1} f(x)+c_{2} g(x)\right) d x=c_{1}(F R) \int_{a}^{b} f(x) d x+c_{2}(F R) \int_{a}^{b} g(x) d x
$$

Also we need.

Lemma 1. ([1]) If $f, g:[a, b] \subseteq \mathbb{R} \rightarrow \mathbb{R}_{\mathscr{F}}$ are fuzzy continuous functions, then the function $F:[a, b] \rightarrow \mathbb{R}_{+}$defined by $F(x):=D(f(x), g(x))$ is continuous on $[a, b]$, and

$$
D\left((F R) \int_{a}^{b} f(x) d x,(F R) \int_{a}^{b} g(x) d x\right) \leq(F R) \int_{a}^{b} D(f(x), g(x)) d x .
$$

For the definition of general fuzzy integral we follow [18] next.

Definition 6. Let $(\Omega, \Sigma, \mu)$ be a complete $\sigma$-finite measure space. We call $F: \Omega \rightarrow R_{\mathscr{F}}$ measurable iff $\forall$ closed $B \subseteq \mathbb{R}$ the function $F^{-1}(B): \Omega \rightarrow[0,1]$ defined by

$$
F^{-1}(B)(w):=\sup _{x \in B} F(w)(x), \text { all } w \in \Omega
$$

is measurable, see [18].

Theorem 5. ([18]) For $F: \Omega \rightarrow \mathbb{R}_{\mathscr{F}}$,

$$
F(w)=\left\{\left(F_{-}^{(r)}(w), F_{+}^{(r)}(w)\right) \mid 0 \leq r \leq 1\right\},
$$

the following are equivalent

(1) $F$ is measurable,

(2) $\forall r \in[0,1], F_{-}^{(r)}, F_{+}^{(r)}$ are measurable.

Following [18], given that for each $r \in[0,1], F_{-}^{(r)}, F_{+}^{(r)}$ are integrable we have that the parametrized representation

$$
\left\{\left(\int_{A} F_{-}^{(r)} d \mu, \int_{A} F_{+}^{(r)} d \mu\right) \mid 0 \leq r \leq 1\right\}
$$

is a fuzzy real number for each $A \in \Sigma$. The last fact leads to,

Definition 7. ([18]) A measurable function $F: \Omega \rightarrow \mathbb{R}_{\mathscr{F}}$,

$$
F(w)=\left\{\left(F_{-}^{(r)}(w), F_{+}^{(r)}(w)\right) \mid 0 \leq r \leq 1\right\}
$$


is integrable if for each $r \in[0,1], F_{ \pm}^{(r)}$ are integrable, or equivalently, if $F_{ \pm}^{(0)}$ are integrable.

In this case, the fuzzy integral of $F$ over $A \in \Sigma$ is defined by

$$
\int_{A} F d \mu:=\left\{\left(\int_{A} F_{-}^{(r)} d \mu, \int_{A} F_{+}^{(r)} d \mu\right) \mid 0 \leq r \leq 1\right\} .
$$

By [18], $F$ is integrable iff $w \rightarrow\|F(w)\|_{\mathscr{F}}$ is real-valued integrable. Here denote

$$
\|u\|_{\mathscr{F}}:=D(u, \widetilde{0}), \forall u \in \mathbb{R}_{\mathscr{F}} .
$$

We need also

Theorem 6. ([18]) Let $F, G: \Omega \rightarrow \mathbb{R}_{\mathscr{F}}$ be integrable. Then

(1) Let $a, b \in \mathbb{R}$, then $a F+b G$ is integrable and for each $A \in \Sigma$,

$$
\int_{A}(a F+b G) d \mu=a \int_{A} F d \mu+b \int_{A} G d \mu
$$

(2) $D(F, G)$ is a real- valued integrable function and for each $A \in \Sigma$,

$$
D\left(\int_{A} F d \mu, \int_{A} G d \mu\right) \leq \int_{A} D(F, G) d \mu .
$$

In particular,

$$
\left\|\int_{A} F d \mu\right\|_{\mathscr{F}} \leq \int_{A}\|F\|_{\mathscr{F}} d \mu \text {. }
$$

Above $\mu$ could be the Lebesgue measure, with all the basic properties valid here too. Basically here we have

$$
\left[\int_{A} F d \mu\right]^{r}=\left[\int_{A} F_{-}^{(r)} d \mu, \int_{A} F_{+}^{(r)} d \mu\right]
$$

i.e.

$$
\left(\int_{A} F d \mu\right)_{ \pm}^{(r)}=\int_{A} F_{ \pm}^{(r)} d \mu, \forall r \in[0,1]
$$

respectively.

\section{Fractional calculus background}

Definition 8. We consider the Prabhakar function (also known as the three parameter Mittag-Leffler function), (see [16], p. 97; [14])

$$
E_{\alpha, \beta}^{\gamma}(z)=\sum_{k=0}^{\infty} \frac{(\gamma)_{k}}{k ! \Gamma(\alpha k+\beta)} z^{k}
$$

where $\Gamma$ is the gamma function; $\alpha, \beta, \gamma \in \mathbb{R}: \alpha, \beta>0, z \in \mathbb{R}$, and $(\gamma)_{k}=\gamma(\gamma+1) \ldots(\gamma+k-1)$. It is $E_{\alpha, \beta}^{0}(z)=\frac{1}{\Gamma(\beta)}$.

Let $a, b \in \mathbb{R}, a<b$ and $x \in[a, b] ; f \in C([a, b])$. Let also $\psi \in C^{1}([a, b])$ which is increasing. The left and right Prabhakar fractional integrals with respect to $\psi$ are defined ([9]) as follows:

$$
\left(e_{\rho, \mu, \omega, a+}^{\gamma ; \psi} f\right)(x)=\int_{a}^{x} \psi^{\prime}(t)(\psi(x)-\psi(t))^{\mu-1} E_{\rho, \mu}^{\gamma}\left[\omega(\psi(x)-\psi(t))^{\rho}\right] f(t) d t
$$


and

$$
\left(e_{\rho, \mu, \omega, b-}^{\gamma ; \psi} f\right)(x)=\int_{x}^{b} \psi^{\prime}(t)(\psi(t)-\psi(x))^{\mu-1} E_{\rho, \mu}^{\gamma}\left[\omega(\psi(t)-\psi(x))^{\rho}\right] f(t) d t,
$$

where $\rho, \mu>0 ; \gamma, \omega \in \mathbb{R}$.

Functions (2) and (3) are continuous, see [9].

Next, additionally, assume that $\psi^{\prime}(x) \neq 0$ over $[a, b]$.

Let $\psi, f \in C^{N}([a, b])$, where $N=\lceil\mu\rceil$, ( $\lceil\cdot\rceil$ is the ceiling of the number $), 0<\mu \notin \mathbb{N}$. We define the $\psi$-Prabhakar-Caputo left and right fractional derivatives of order $\mu$ ([9]) as follows $(x \in[a, b])$ :

$$
\left({ }^{C} D_{\rho, \mu, \omega, a+}^{\gamma ; \psi} f\right)(x)=\int_{a}^{x} \psi^{\prime}(t)(\psi(x)-\psi(t))^{N-\mu-1} E_{\rho, N-\mu}^{-\gamma}\left[\omega(\psi(x)-\psi(t))^{\rho}\right]\left(\frac{1}{\psi^{\prime}(t)} \frac{d}{d t}\right)^{N} f(t) d t
$$

and

$$
\left({ }^{C} D_{\rho, \mu, \omega, b-}^{\gamma ; \psi} f\right)(x)=(-1)^{N} \int_{x}^{b} \psi^{\prime}(t)(\psi(t)-\psi(x))^{N-\mu-1} E_{\rho, N-\mu}^{-\gamma}\left[\omega(\psi(t)-\psi(x))^{\rho}\right]\left(\frac{1}{\psi^{\prime}(t)} \frac{d}{d t}\right)^{N} f(t) d t
$$

One can write these (see (4), (5)) as

$$
\left({ }^{C} D_{\rho, \mu, \omega, a+}^{\gamma ; \psi} f\right)(x)=\left(e_{\rho, N-\mu, \omega, a+}^{-\gamma ; \psi} f_{\psi}^{[N]}\right)(x)
$$

and

$$
\left({ }^{C} D_{\rho, \mu, \omega, b-}^{\gamma ; \psi} f\right)(x)=(-1)^{N}\left(e_{\rho, N-\mu, \omega, b-}^{-\gamma ; \psi} f_{\psi}^{[N]}\right)(x),
$$

where

$$
f_{\psi}^{[N]}(x)=f_{\psi}^{(N)} f(x):=\left(\frac{1}{\psi^{\prime}(x)} \frac{d}{d x}\right)^{N} f(x)
$$

$\forall x \in[a, b]$.

Functions (6) and (7) are continuous on $[a, b]$. We will apply (2)-(8) for $\psi(x)=x$. We need,

Definition 9. Here we use the multivariate analogue of generalized Mittag-Leffler function, see [20], defined for $\lambda, \gamma_{j}, \rho_{j}, z_{j} \in \mathbb{C}, \operatorname{Re}\left(\rho_{j}\right)>0(j=1, \ldots, m)$ in terms of a multiple series of the form:

$$
E_{\left(\rho_{j}\right), \lambda}^{\left(\gamma_{j}\right)}\left(z_{1}, \ldots, z_{m}\right)=E_{\left(\rho_{1}, \ldots, \rho_{m}\right), \lambda}^{\left(\gamma_{1}, \ldots, \gamma_{m}\right)}\left(z_{1}, \ldots, z_{m}\right)=\sum_{k_{1}, \ldots, k_{m}=0}^{\infty} \frac{\left(\gamma_{1}\right)_{k_{1}} \ldots\left(\gamma_{m}\right)_{k_{m}}}{\Gamma\left(\lambda+\sum_{j=1}^{m} k_{j} \rho_{j}\right)} \frac{z_{1}^{k_{1}} \ldots z_{m}^{k_{m}}}{k_{1} ! \ldots k_{m} !}
$$

where $\left(\gamma_{j}\right)_{k_{j}}$ is the Pochhammer symbol, $\Gamma$ is the gamma function. This is a special case of the generalized Lauricella series in several variables, see [21], p. 454 and [23].

By [22], p. 157, (9) converges for $\operatorname{Re}\left(\rho_{j}\right)>0, j=1, \ldots, m$. In what follows we will use the particular case of $E_{(\rho, \ldots, \rho), \lambda}^{\left(\gamma_{1}, \ldots, \gamma_{m}\right)}\left[\omega_{1} t^{\rho}, \ldots, \omega_{m} t^{\rho}\right]$, denoted by $E_{(\rho), \lambda}^{\left(\gamma_{j}\right)}\left[\omega_{1} t^{\rho}, \ldots, \omega_{m} t^{\rho}\right]$, where $0<\rho<1, t \geq 0, \lambda>0, \gamma_{j} \in \mathbb{R}$ with $\left(\gamma_{j}\right)_{k_{j}}:=\gamma_{j}\left(\gamma_{j}+1\right) \ldots\left(\gamma_{j}+k_{j}-1\right), \omega_{j} \in \mathbb{R}-\{0\}$, for $j=1, \ldots$, m. Let $f \in C^{1}([a, b])$, we define the following Caputo 
type generalized left fractional derivative with non singular kernel of order $\rho$, as

$$
D_{a *}^{\rho} f(x):=\underset{\left(\gamma_{j}\right)\left(\omega_{j}\right)}{C A} D_{a *}^{\rho, \lambda} f(x):=\frac{A(\rho)}{1-\rho} \int_{a}^{x} E_{(\rho), \lambda}^{\left(\gamma_{j}\right)}\left[\frac{-\omega_{1} \rho}{1-\rho}(x-t)^{\rho}, \ldots, \frac{-\omega_{m} \rho}{1-\rho}(x-t)^{\rho}\right] f^{\prime}(t) d t, x \in[a, b],
$$

where $A(\rho)$ is a normalizing constant.

Let now $f \in C^{n+1}([a, b]), n \in \mathbb{Z}_{+}$.

We define the Caputo type generalized left fractional derivative with non singular kernel of order $n+\rho$, as

$$
\begin{gathered}
D_{a *}^{n+\rho} f(x):=\begin{array}{c}
C A \\
\left(\gamma_{j}\right)\left(\omega_{j}\right)
\end{array} D_{a *}^{n+\rho, \lambda} f(x):= \\
\frac{A(\rho)}{1-\rho} \int_{a}^{x} E_{(\rho), \lambda}^{\left(\gamma_{j}\right)}\left[\frac{-\omega_{1} \rho}{1-\rho}(x-t)^{\rho}, \ldots, \frac{-\omega_{m} \rho}{1-\rho}(x-t)^{\rho}\right] f^{(n+1)}(t) d t, x \in[a, b] .
\end{gathered}
$$

Similarly, we define the Caputo type generalized right fractional derivative with non singular kernel of order $\rho$, as

$$
\begin{gathered}
D_{b-}^{\rho} f(x):=\begin{array}{l}
C A \\
\left(\gamma_{j}\right)\left(\omega_{j}\right)
\end{array} D_{b-}^{\rho, \lambda} f(x):= \\
\frac{-A(\rho)}{1-\rho} \int_{x}^{b} E_{(\rho), \lambda}^{\left(\gamma_{j}\right)}\left[\frac{-\omega_{1} \rho}{1-\rho}(t-x)^{\rho}, \ldots, \frac{-\omega_{m} \rho}{1-\rho}(t-x)^{\rho}\right] f^{\prime}(t) d t, x \in[a, b] .
\end{gathered}
$$

And, for $f \in C^{n+1}([a, b]), n \in \mathbb{Z}_{+}$, we define

$$
\begin{gathered}
D_{b-}^{n+\rho} f(x):=\begin{array}{c}
C A \\
\left(\gamma_{j}\right)\left(\omega_{j}\right)
\end{array} D_{b-}^{n+\rho, \lambda} f(x):= \\
(-1)^{n+1} \frac{A(\rho)}{1-\rho} \int_{x}^{b} E_{(\rho), \lambda}^{\left(\gamma_{j}\right)}\left[\frac{-\omega_{1} \rho}{1-\rho}(t-x)^{\rho}, \ldots, \frac{-\omega_{m} \rho}{1-\rho}(t-x)^{\rho}\right] f^{(n+1)}(t) d t,
\end{gathered}
$$

$x \in[a, b]$.

The above derivatives generalize the Atangana-Baleanu fractional derivative, see [10], also see next.

We mention the following non-singular kernel fractional derivatives.

Definition 10. Let $f \in C^{1}([a, b]), a<b, \alpha \in(0,1)$, the Atangana-Baleanu left fractional derivative ([10]) is given by

$$
\left({ }^{A B C} D_{a+}^{\alpha} f\right)(x)=\frac{B(\alpha)}{1-\alpha} \int_{a}^{x} E_{\alpha}\left(-\alpha \frac{(x-t)^{\alpha}}{1-\alpha}\right) f^{\prime}(t) d t
$$

$\forall x \in[a, b]$, where $B(\alpha)$ is a normalizing constant and $E_{\alpha}$ is the one parameter Mittag-Leffler function:

$$
E_{\alpha}(x)=\sum_{k=0}^{\infty} \frac{x^{k}}{\Gamma(\alpha k+1)}, x \in \mathbb{R}
$$

which is an entire function converging for all $x \in \mathbb{R}$. The right side fractional derivative is defined by

$$
\left({ }^{A B C} D_{b-}^{\alpha} f\right)(x)=-\frac{B(\alpha)}{1-\alpha} \int_{x}^{b} E_{\alpha}\left(-\alpha \frac{(t-x)^{\alpha}}{1-\alpha}\right) f^{\prime}(t) d t
$$


$\forall x \in[a, b]$. Let now $f \in C^{n+1}([a, b]), n \in \mathbb{Z}_{+}$. We define more generally, the left side fractional derivative:

$$
\left({ }^{A B C} D_{a+}^{n+\alpha} f\right)(x)=\frac{B(\alpha)}{1-\alpha} \int_{a}^{x} E_{\alpha}\left(-\alpha \frac{(x-t)^{\alpha}}{1-\alpha}\right) f^{(n+1)}(t) d t
$$

$\forall x \in[a, b]$, and the right side one:

$$
\left({ }^{A B C} D_{b-}^{n+\alpha} f\right)(x)=(-1)^{n+1} \frac{B(\alpha)}{1-\alpha} \int_{x}^{b} E_{\alpha}\left(-\alpha \frac{(t-x)^{\alpha}}{1-\alpha}\right) f^{(n+1)}(t) d t
$$

$\forall x \in[a, b]$. Fractional derivatives (14), (16), (17), (18) generalize the following Caputo-Fabrizio non-singular kernel fractional derivatives, see [12], [19].

Definition 11. Let $f \in C^{1}([a, b]), \alpha \in(0,1)$, the Caputo-Fabrizio ([12], [19]) corresponding fractional derivatives have as follows:

$$
{ }^{C F} D_{a+}^{\alpha} f(x)=\frac{1}{1-\alpha} \int_{a}^{x} \exp \left(-\frac{\alpha}{1-\alpha}(x-t)\right) f^{\prime}(t) d t
$$

and

$$
{ }^{C F} D_{b-}^{\alpha} f(x)=-\frac{1}{1-\alpha} \int_{x}^{b} \exp \left(-\frac{\alpha}{1-\alpha}(t-x)\right) f^{\prime}(t) d t,
$$

$\forall x \in[a, b]$. When $f \in C^{n+1}([a, b]), n \in \mathbb{Z}_{+}$, we have

$$
{ }^{C F} D_{a+}^{n+\alpha} f(x)=\frac{1}{1-\alpha} \int_{a}^{x} \exp \left(-\frac{\alpha}{1-\alpha}(x-t)\right) f^{(n+1)}(t) d t,
$$

and

$$
{ }^{C F} D_{b-}^{n+\alpha} f(x)=\frac{(-1)^{n+1}}{1-\alpha} \int_{x}^{b} \exp \left(-\frac{\alpha}{1-\alpha}(t-x)\right) f^{(n+1)}(t) d t
$$

$\forall x \in[a, b]$.

Finally we give

Definition 12. Let $f \in C^{1}([a, b]), \alpha \in(0,1)$, the Atangana-Gomez ([11]) corresponding fractional derivatives have as follows:

$$
{ }^{A G} D_{a+}^{\alpha} f(x)=\frac{A G(\alpha)}{1-\alpha} \int_{a}^{x} \exp \left(-\frac{\alpha}{1-\alpha}(x-t)^{2}\right) f^{\prime}(t) d t
$$

and

$$
{ }^{A G} D_{b-}^{\alpha} f(x)=-\frac{A G(\alpha)}{1-\alpha} \int_{x}^{b} \exp \left(-\frac{\alpha}{1-\alpha}(x-t)^{2}\right) f^{\prime}(t) d t
$$

$\forall x \in[a, b]$. When $f \in C^{n+1}([a, b]), n \in \mathbb{Z}_{+}$, we have

$$
{ }^{A G} D_{a+}^{n+\alpha} f(x)=\frac{A G(\alpha)}{1-\alpha} \int_{a}^{x} \exp \left(-\frac{\alpha}{1-\alpha}(x-t)^{2}\right) f^{(n+1)}(t) d t
$$

and

$$
{ }^{A G} D_{b-}^{n+\alpha} f(x)=\frac{(-1)^{n+1} A G(\alpha)}{1-\alpha} \int_{x}^{b} \exp \left(-\frac{\alpha}{1-\alpha}(x-t)^{2}\right) f^{(n+1)}(t) d t,
$$

$\forall x \in[a, b]$, where $A G(\alpha)$ is a normalization constant.

In [6], Chapter 34, we had introduced and studied the left and right fuzzy fractional Riemann-Liouville integrals and the left and right fuzzy fractional Caputo derivatives. 
In this article we define the corresponding to section 2, fuzzy fractional entities, and we study some of their basic properties. Furthermore we derive some related fuzzy fractional inequalities of Opial and Hardy types.

\section{Main Results}

We need

Definition 13. Let the kernel $k:[0, b-a] \rightarrow \mathbb{R}_{+} ; a<b$, be an integrable function, and let $v \geq 0$ with $n=\lceil v\rceil \in \mathbb{Z}_{+}$, and $f \in C_{\mathscr{F}}^{n}([a, b])$.

We define the generalized fuzzy fractional derivatives of order $v$ (or integrals $v=0$ ) left and right; respectively, as follows:

$$
{ }^{k} D_{a+}^{\mathscr{F}} f(x):=\int_{a}^{x} k(x-t) \odot f(n)(t) d t
$$

and

$$
{ }^{k} D_{b-}^{\mathscr{F}} f(x):=(-1)^{n} \odot \int_{x}^{b} k(t-x) \odot f^{(n)}(t) d t,
$$

$\forall x \in[a, b]$. Furthermore, when $g \in C^{n}([a, b])$ we define

$$
{ }^{k} D_{a+} g(x):=\int_{a}^{x} k(x-t) g^{(n)}(t) d t,
$$

and

$$
{ }^{k} D_{b-} g(x):=(-1)^{n} \int_{x}^{b} k(t-x) g^{(n)}(t) d t,
$$

$\forall x \in[a, b], n \in \mathbb{Z}_{+}$, the corresponding real fractional derivatives and integrals.

We make,

Remark. We have that (by Definition 7)

$$
\begin{gathered}
{ }^{k} D_{a+}^{\mathscr{F}} f(x)=\int_{a}^{x} k(x-t) \odot f^{(n)}(t) d t=\left\{\left(\int_{a}^{x} k(x-t)\left(f^{(n)}\right)_{-}^{(r)}(t) d t, \int_{a}^{x} k(x-t)\left(f^{(n)}\right)_{+}^{(r)}(t) d t\right) \mid 0 \leq r \leq 1\right\} \\
=\left\{\left(\int_{a}^{x} k(x-t)\left(f_{-}^{(r)}\right)^{(n)}(t) d t, \int_{a}^{x} k(x-t)\left(f_{+}^{(r)}\right)^{(n)}(t) d t\right) \mid 0 \leq r \leq 1\right\} .
\end{gathered}
$$

So, we obtain

$$
\left[{ }^{k} D_{a+}^{\mathscr{F}} f(x)\right]^{r}=\left[\int_{a}^{x} k(x-t)\left(f_{-}^{(r)}\right)^{(n)}(t) d t, \int_{a}^{x} k(x-t)\left(f_{+}^{(r)}\right)^{(n)}(t) d t\right]
$$

$0 \leq r \leq 1$. That is

$$
\left({ }^{k} D_{a+}^{\mathscr{F}} f(x)\right)_{ \pm}^{(r)}=\int_{a}^{x} k(x-t)\left(f_{ \pm}^{(r)}\right)^{(n)}(t) d t=\left({ }^{k} D_{a+}\left(f_{ \pm}^{(r)}\right)\right)(x)
$$

i.e. we get

$$
\left({ }^{k} D_{a+}^{\mathscr{F}} f\right)_{ \pm}^{(r)}={ }^{k} D_{a+}\left(f_{ \pm}^{(r)}\right), \forall r \in[0,1]
$$

Similarly, we obtain that

$$
{ }^{k} D_{b-}^{\mathscr{F}} f(x)=(-1)^{n} \odot \int_{x}^{b} k(t-x) \odot f^{(n)}(t) d t
$$




$$
\begin{aligned}
& =\left\{\left((-1)^{n} \int_{x}^{b} k(t-x)\left(f^{(n)}\right)_{-}^{(r)}(t) d t,(-1)^{n} \int_{x}^{b} k(t-x)\left(f^{(n)}\right)_{+}^{(r)}(t) d t\right) \mid 0 \leq r \leq 1\right\} \\
& =\left\{\left((-1)^{n} \int_{x}^{b} k(t-x)\left(f_{-}^{(r)}\right)^{(n)}(t) d t,(-1)^{n} \int_{x}^{b} k(t-x)\left(f_{+}^{(r)}\right)^{(n)}(t) d t\right) \mid 0 \leq r \leq 1\right\} .
\end{aligned}
$$

So, we obtain

$$
\left[{ }^{k} D_{b-}^{\mathscr{F}} f(x)\right]^{r}=\left[(-1)^{n} \int_{x}^{b} k(t-x)\left(f_{-}^{(r)}\right)^{(n)}(t) d t,(-1)^{n} \int_{x}^{b} k(t-x)\left(f_{+}^{(r)}\right)^{(n)}(t) d t\right],
$$

$0 \leq r \leq 1$. That is

$$
\left({ }^{k} D_{b-}^{\mathscr{F}} f(x)\right)_{ \pm}^{(r)}=(-1)^{n} \int_{x}^{b} k(t-x)\left(f_{ \pm}^{(r)}\right)^{(n)}(t) d t=\left({ }^{k} D_{b-}\left(f_{ \pm}^{(r)}\right)\right)(x)
$$

i.e. we get

$$
\left({ }^{k} D_{b-}^{\mathscr{F}} f\right)_{ \pm}^{(r)}={ }^{k} D_{b-}\left(f_{ \pm}^{(r)}\right), \forall r \in[0,1]
$$

Another important matter follows: It holds that $\lim _{y \rightarrow x} \int_{x-a}^{y-a} k(z) d z=\lim _{y \rightarrow x} \int_{0}^{b-a} \chi_{[x-a, y-a]}(z) k(z) d z=0$, by the integrability of $k$ and dominated convergence theorem, where $x, y \in[a, b], y \geq x ; \chi$ is the characteristic function.

Similarly, it holds that $\lim _{y \rightarrow x} \int_{b-x}^{b-y} k(z) d z=0$, where $x, y \in[a, b], y \leq x$. We give

Theorem 7. All as in Definition 13. ${ }^{k} D_{a+}^{\mathscr{F}} f(x)$ is fuzzy continuous in $x \in[a, b]$.

Proof. Without loss of generality we may assume $a \leq x \leq y \leq b$, that is $0 \leq x-a \leq y-a \leq b-a$. So, we have

$$
\begin{gathered}
D\left({ }^{k} D_{a+}^{\mathscr{F}} f(x),{ }^{k} D_{a+}^{\mathscr{F}} f(y)\right)=D\left(\int_{a}^{x} k(x-t) \odot f^{(n)}(t) d t, \int_{a}^{y} k(y-t) \odot f^{(n)}(t) d t\right) \\
=D\left(\int_{0}^{x-a} k(z) \odot f^{(n)}(x-z) d z, \int_{0}^{y-a} k(z) \odot f^{(n)}(y-z) d z\right)=D\left(\int_{0}^{x-a} k(z) \odot f^{(n)}(x-z) d z,\right. \\
\left.\leq \int_{0}^{x-a} k(z) \odot f^{(n)}(y-z) d z \oplus \int_{x-a}^{y-a} k(z) \odot f^{(n)}(y-z) d z\right) \\
\left.\leq \int_{0}^{x-a} k(z) \odot f^{(n)}(x-z) d z, \int_{0}^{x-a} k(z) \odot f^{(n)}(y-z) d z\right)+D\left(\int_{x-a}^{y-a} k(z) \odot f^{(n)}(y-z) d z, \tilde{0}\right) \\
\leq \int_{0}^{x-a} k(z) D\left(f^{(n)}(x-z), f^{(n)}(y-z)\right) d z+\int_{x-a}^{y-a} k(z) D\left(f^{(n)}(y-z), \widetilde{0}\right) d z \\
\leq\left(\int_{0}^{x-a} k(z) d z\right) \omega_{1}^{(\mathscr{F})}\left(f^{(n)}, y-x\right)+D^{*}\left(f^{(n)}, \widetilde{0}\right) \int_{x-a}^{y-a} k(z) d z \\
\leq\left\{\left(\int_{0}^{b-a} k(z) d z\right) \omega_{1}^{(\mathscr{F})}\left(f^{(n)}, y-x\right)+D^{*}\left(f^{(n)}, \widetilde{0}\right) \int_{x-a}^{y-a} k(z) d z\right\} \rightarrow 0,
\end{gathered}
$$

as $y \rightarrow x$, by noticing that $f^{(n)} \in C_{\mathscr{F}}^{U}([a, b])$.

We also present

Theorem 8. All as in Definition 13. ${ }^{k} D_{b-}^{\mathscr{F}} f(x)$ is fuzzy continuous in $x \in[a, b]$. 
Proof. Without loss of generality we suppose $a \leq y \leq x \leq b$, that is $0 \leq b-x \leq b-y \leq b-a$. So, we have

$$
\begin{gathered}
D\left({ }^{k} D_{b-}^{\mathscr{F}} f(x),{ }^{k} D_{b-}^{\mathscr{F}} f(y)\right)=D\left((-1)^{n} \odot \int_{x}^{b} k(t-x) \odot f^{(n)}(t) d t,(-1)^{n} \odot \int_{y}^{b} k(t-y) \odot f^{(n)}(t) d t\right) \\
=D\left(\int_{x}^{b} k(t-x) \odot f^{(n)}(t) d t, \int_{y}^{b} k(t-y) \odot f^{(n)}(t) d t\right) \\
=D\left(\int_{0}^{b-x} k(z) \odot f^{(n)}(z+x) d z, \int_{0}^{b-y} k(z) \odot f^{(n)}(z+y) d z\right) \\
=D\left(\int_{0}^{b-x} k(z) \odot f^{(n)}(z+x) d z\right. \\
\leq D\left(\int_{0}^{b-x} k(z) \odot f^{(n)}(z+x) d z, \int_{0}^{b-x} k(z) \odot f^{(n)}(z+y) d z\right)+D\left(\int_{b-x}^{b-y} k(z) \odot f^{(n)}(z+y) d z, \widetilde{0}\right) \\
\leq \int_{0}^{b-x} k(z) D\left(f^{(n)}(z+x), f^{(n)}(z+y)\right) d z+\int_{b-x}^{b-y} k(z) D\left(f^{(n)}(z+y), \widetilde{0}\right) d z \\
\leq\left(\int_{0}^{b-x} k(z) d z\right) \omega_{1}^{(\mathscr{F})}\left(f^{(n)}, x-y\right)+D^{*}\left(f^{(n)}, \widetilde{0}\right) \int_{b-x}^{b-y} k(z) d z \\
\leq\left\{\left(\int_{0}^{b-a} k(z) d z\right) \omega_{1}^{(\mathscr{F})}\left(f^{(n)}, x-y\right)+D^{*}\left(f^{(n)}, \widetilde{0}\right) \int_{b-x}^{b-y} k(z) d z\right\} \rightarrow 0,
\end{gathered}
$$

as $y \rightarrow x$, by noticing that $f^{(n)} \in C_{\mathscr{F}}^{U}([a, b])$.

We present the following left Opial type general inequality.

Theorem 9. Let $p, q>1: \frac{1}{p}+\frac{1}{q}=1$. All here as in Definition 13. Assume that $k \in L_{p}(0, b-a)$. Then

$$
\int_{a}^{x} D\left({ }^{k} D_{a+}^{\mathscr{F}} f(w), \widetilde{0}\right) D\left(f^{(n)}(w), \widetilde{0}\right) d w \leq 2^{-\frac{1}{q}}\left(\int_{a}^{x}\left(\int_{a}^{w} k(w-t)^{p} d t\right) d w\right)^{\frac{1}{p}}\left(\int_{a}^{x}\left(D\left(f^{(n)}(w), \widetilde{0}\right)\right)^{q} d w\right)^{\frac{2}{q}}
$$

$\forall x \in[a, b]$.

Proof. We have that

$$
D\left({ }^{k} D_{a+}^{\mathscr{F}} f(w), \widetilde{0}\right) \stackrel{(27)}{=} D\left(\int_{a}^{x} k(x-t) \odot f^{(n)}(t) d t, \widetilde{0}\right) \leq \int_{a}^{x} k(x-t) D\left(f^{(n)}(t), \widetilde{0}\right) d t \leq
$$

(by Hölder's inequality)

$$
\left(\int_{a}^{x} k(x-t)^{p} d t\right)^{\frac{1}{p}}\left(\int_{a}^{x}\left(D\left(f^{(n)}(t), \widetilde{0}\right)\right)^{q} d t\right)^{\frac{1}{q}}
$$

Call

$$
\rho(x):=\int_{a}^{x}\left(D\left(f^{(n)}(t), \widetilde{0}\right)\right)^{q} d t, \rho(a)=0 .
$$

Then

$$
\rho^{\prime}(x)=\left(D\left(f^{(n)}(x), \widetilde{0}\right)\right)^{q} \geq 0,
$$


and

$$
\left(\rho^{\prime}(x)\right)^{\frac{1}{q}}=\left(D\left(f^{(n)}(x), \widetilde{0}\right)\right) \geq 0, \forall x \in[a, b]
$$

Consequently, we get

$$
\left({ }^{k} D_{a+}^{\mathscr{F}} f(w), \widetilde{0}\right) D\left(f^{(n)}(w), \widetilde{0}\right) \leq\left(\int_{a}^{w} k(w-t)^{p} d t\right)^{\frac{1}{p}}\left(\rho(w) \rho^{\prime}(w)\right)^{\frac{1}{q}}
$$

$\forall w \in[a, b]$. Then, by applying again Hölder's inequality:

$$
\begin{gathered}
\int_{a}^{x} D\left({ }^{k} D_{a+}^{\mathscr{F}} f(w), \widetilde{0}\right) D\left(f^{(n)}(w), \widetilde{0}\right) d w \leq \int_{a}^{x}\left[\left(\int_{a}^{w} k(w-t)^{p} d t\right)^{\frac{1}{p}}\left(\rho(w) \rho^{\prime}(w)\right)^{\frac{1}{q}}\right] d w \\
\leq\left(\int_{a}^{x}\left(\int_{a}^{w} k(w-t)^{p} d t\right) d w\right)^{\frac{1}{p}}\left(\int_{a}^{x} \rho(w) d \rho(w)\right)^{\frac{1}{q}}=\left(\int_{a}^{x}\left(\int_{a}^{w} k(w-t)^{p} d t\right) d w\right)^{\frac{1}{p}}\left(\frac{\rho^{2}(x)}{2}\right)^{\frac{1}{q}} \\
=2^{-\frac{1}{q}}\left(\int_{a}^{x}\left(\int_{a}^{w} k(w-t)^{p} d t\right) d w\right)^{\frac{1}{p}}\left(\int_{a}^{x}\left(D\left(f^{(n)}(w), \widetilde{0}\right)\right)^{q} d w\right)^{\frac{2}{q}}
\end{gathered}
$$

proving the claim.

The right side corresponding fractional Opial type general inequality follows:

Theorem 10. All as in Theorem 9. Then

$$
\begin{gathered}
\int_{x}^{b} D\left({ }^{k} D_{b-}^{\mathscr{F}} f(w), \widetilde{0}\right) D\left(f^{(n)}(w), \widetilde{0}\right) d w \leq \\
2^{-\frac{1}{q}}\left(\int_{x}^{b}\left(\int_{w}^{b} k(t-w)^{p} d t\right) d w\right)^{\frac{1}{p}}\left(\int_{x}^{b}\left(D\left(f^{(n)}(w), \widetilde{0}\right)\right)^{q} d w\right)^{\frac{2}{q}}
\end{gathered}
$$

$\forall x \in[a, b]$.

Proof. As similar to the proof of Theorem 9 is omitted.

Next follow general left and right fractional Hardy type inequalities.

Theorem 11. All as in Theorem 9. Then

(1)

$$
\left\|D\left({ }^{k} D_{a+}^{\mathscr{F}} f(\cdot), \widetilde{0}\right)\right\|_{q,[a, b]} \leq\left(\int_{a}^{b}\left(\int_{a}^{x} k(x-t)^{p} d t\right)^{\frac{q}{p}} d x\right)^{\frac{1}{q}}\left\|D\left(f^{(n)}(\cdot), \widetilde{0}\right)\right\|_{q,[a, b]}
$$

(2)

$$
\left\|D\left({ }^{k} D_{b-}^{\mathscr{F}} f(\cdot), \widetilde{0}\right)\right\|_{q,[a, b]} \leq\left(\int_{a}^{b}\left(\int_{x}^{b} k(t-x)^{p} d t\right)^{\frac{q}{p}} d x\right)^{\frac{1}{q}}\left\|D\left(f^{(n)}(\cdot), \widetilde{0}\right)\right\|_{q,[a, b]}
$$

Proof. (1) By (44) we obtain

$$
D\left({ }^{k} D_{a+}^{\mathscr{F}} f(x), \widetilde{0}\right) \leq\left(\int_{a}^{x} k(x-t)^{p} d t\right)^{\frac{1}{p}}\left\|D\left(f^{(n)}(\cdot), \widetilde{0}\right)\right\|_{q,[a, b]}
$$


Hence we have

$$
D\left({ }^{k} D_{a+}^{\mathscr{F}} f(x), \widetilde{0}\right)^{q} \leq\left(\int_{a}^{x} k(x-t)^{p} d t\right)^{\frac{q}{p}}\left\|D\left(f^{(n)}(\cdot), \widetilde{0}\right)\right\|_{q,[a, b]}^{q}
$$

and

$$
\int_{a}^{b} D\left({ }^{k} D_{a+}^{\mathscr{F}} f(x), \widetilde{0}\right)^{q} d x \leq\left(\int_{a}^{b}\left(\int_{a}^{x} k(x-t)^{p} d t\right)^{\frac{q}{p}} d x\right)\left\|D\left(f^{(n)}(\cdot), \widetilde{0}\right)\right\|_{q,[a, b]}^{q}
$$

Therefore it holds

$$
\left(\int_{a}^{b} D\left({ }^{k} D_{a+}^{\mathscr{F}} f(x), \widetilde{0}\right)^{q} d x\right)^{\frac{1}{q}} \leq\left(\int_{a}^{b}\left(\int_{a}^{x} k(x-t)^{p} d t\right)^{\frac{q}{p}} d x\right)^{\frac{1}{q}}\left\|D\left(f^{(n)}(\cdot), \widetilde{0}\right)\right\|_{q,[a, b]}
$$

proving the claim.

(2) the proof of (52) is similar and thus it is omitted.

One can derive a vast wealth of fuzzy fractional inequalities based on author's monographs [4]-[8].

Next we write down Definition 8, when $\psi(x)=x$.

Definition 14. Let $f \in C([a, b])$. The left and right Prabhakar fractional integrals are defined as follows:

$$
\left(e_{\rho, \mu, \omega, a+}^{\gamma} f\right)(x)=\int_{a}^{x}(x-t)^{\mu-1} E_{\rho, \mu}^{\gamma}\left[\omega(x-t)^{\rho}\right] f(t) d t
$$

and

$$
\left(e_{\rho, \mu, \omega, b-}^{\gamma} f\right)(x)=\int_{x}^{b}(t-x)^{\mu-1} E_{\rho, \mu}^{\gamma}\left[\omega(t-x)^{\rho}\right] f(t) d t
$$

where $\rho, \mu>0 ; \gamma, \omega \in \mathbb{R} ; \forall x \in[a, b]$. Functions (57) and (58) are continuous, see [9].

Let $\lceil\mu\rceil=N, f \in C^{N}([a, b]), 0<\mu \notin \mathbb{N}$. We define the Prabhakar-Caputo left and right fractional derivatives of order $\mu$ as follows $(x \in[a, b])$ :

$$
\left({ }^{C} D_{\rho, \mu, \omega, a+}^{\gamma} f\right)(x)=\int_{a}^{x}(x-t)^{N-\mu-1} E_{\rho, N-\mu}^{-\gamma}\left[\omega(x-t)^{\rho}\right] f^{(N)}(t) d t
$$

and

$$
\left({ }^{C} D_{\rho, \mu, \omega, b-}^{\gamma} f\right)(x)=(-1)^{N} \int_{x}^{b}(t-x)^{N-\mu-1} E_{\rho, N-\mu}^{-\gamma}\left[\omega(t-x)^{\rho}\right] f^{(N)}(t) d t,
$$

that is the continuous functions on $[a, b]$ :

$$
\left({ }^{C} D_{\rho, \mu, \omega, a+}^{\gamma} f\right)(x)=\left(e_{\rho, N-\mu, \omega, a+}^{-\gamma} f^{(N)}\right)(x),
$$

and

$$
\left({ }^{C} D_{\rho, \mu, \omega, b-}^{\gamma} f\right)(x)=(-1)^{N}\left(e_{\rho, N-\mu, \omega, b-}^{-\gamma} f^{(N)}\right)(x)
$$

$\forall x \in[a, b]$.

Based on section 2 real valued fractional integrals and derivatives (see also Definition 14) we define the corresponding fuzzy valued fractional integrals and derivatives. We give, 
Definition 15. Let $f \in C_{\mathscr{F}}([a, b])$. The fuzzy left and right Prabhakar fractional integrals are defined as follows:

$$
\left(e_{\rho, \mu, \omega, a+}^{\gamma, \mathscr{F}} f\right)(x)=\int_{a}^{x}(x-t)^{\mu-1} E_{\rho, \mu}^{\gamma}\left[\omega(x-t)^{\rho}\right] \odot f(t) d t
$$

and

$$
\left(e_{\rho, \mu, \omega, b-}^{\gamma, \mathscr{F}} f\right)(x)=\int_{x}^{b}(t-x)^{\mu-1} E_{\rho, \mu}^{\gamma}\left[\omega(t-x)^{\rho}\right] \odot f(t) d t
$$

where $\rho, \mu>0 ; \gamma, \omega \in \mathbb{R}_{+} ; \forall x \in[a, b]$. Functions (63) and (64) are fuzzy continuous, see Theorems 7 , 8.

Let $\lceil\mu\rceil=N, f \in C_{\mathscr{F}}^{N}([a, b]), 0<\mu \notin \mathbb{N}$. We define the fuzzy Prabhakar-Caputo left and right fractional derivatives of order $\mu$ as follows $(x \in[a, b])$ :

$$
\left({ }^{C} D_{\rho, \mu, \omega, a+}^{\gamma, \mathscr{F}} f\right)(x)=\int_{a}^{x}(x-t)^{N-\mu-1} E_{\rho, N-\mu}^{-\gamma}\left[\omega(x-t)^{\rho}\right] \odot f^{(N)}(t) d t
$$

and

$$
\left({ }^{C} D_{\rho, \mu, \omega, b-}^{\gamma, \mathscr{F}} f\right)(x)=(-1)^{N} \odot \int_{x}^{b}(t-x)^{N-\mu-1} E_{\rho, N-\mu}^{-\gamma}\left[\omega(t-x)^{\rho}\right] \odot f^{(N)}(t) d t
$$

that is the fuzzy continuous functions on $[a, b]$ :

$$
\left({ }^{C} D_{\rho, \mu, \omega, a+}^{\gamma, \mathscr{F}} f\right)(x)=\left(e_{\rho, N-\mu, \omega, a+}^{-\gamma, \mathscr{F}} f^{(N)}\right)(x)
$$

and

$$
\left({ }^{C} D_{\rho, \mu, \omega, b-}^{\gamma, \mathscr{F}} f\right)(x)=(-1)^{N} \odot\left(e_{\rho, N-\mu, \omega, b-}^{-\gamma, \mathscr{F}} f^{(N)}\right)(x)
$$

$\forall x \in[a, b]$.

We need,

Definition 16. Here we use the multivariate analogue of generalized Mittag-Leffler function, see [20], defined for $\lambda, \gamma_{j}, \rho_{j}, z_{j} \in \mathbb{C}, \operatorname{Re}\left(\rho_{j}\right)>0(j=1, \ldots, m)$ in terms of a multiple series of the form:

$$
E_{\left(\rho_{j}\right), \lambda}^{\left(\gamma_{j}\right)}\left(z_{1}, \ldots, z_{m}\right)=E_{\left(\rho_{1}, \ldots, \rho_{m}\right), \lambda}^{\left(\gamma_{1}, \ldots, \gamma_{m}\right)}\left(z_{1}, \ldots, z_{m}\right)=\sum_{k_{1}, \ldots, k_{m}=0}^{\infty} \frac{\left(\gamma_{1}\right)_{k_{1}} \ldots\left(\gamma_{m}\right)_{k_{m}}}{\Gamma\left(\lambda+\sum_{j=1}^{m} k_{j} \rho_{j}\right)} \frac{z_{1}^{k_{1}} \ldots z_{m}^{k_{m}}}{k_{1} ! \ldots k_{m} !}
$$

where $\left(\gamma_{j}\right)_{k_{j}}$ is the Pochhammer symbol and $\Gamma$ is the gamma function.

In what follows we will use the particular case of $E_{(\rho, \ldots, \rho), \lambda}^{\left(\gamma_{1}, \ldots, \gamma_{m}\right)}\left[\omega_{1} t^{\rho}, \ldots, \omega_{m} t^{\rho}\right]$, denoted by $E_{(\rho), \lambda}^{\left(\gamma_{j}\right)}\left[\omega_{1} t^{\rho}, \ldots, \omega_{m} t^{\rho}\right]$, where $0<\rho<1, t \geq 0, \lambda>0, \gamma_{j} \in \mathbb{R}_{+}$with $\left(\gamma_{j}\right)_{k_{j}}:=\gamma_{j}\left(\gamma_{j}+1\right) \ldots\left(\gamma_{j}+k_{j}-1\right), \omega_{j} \in \mathbb{R}_{+}-\{0\}$, for $j=1, \ldots, m$. Let $f \in C_{\mathscr{F}}^{1}([a, b])$, we define the following fuzzy Caputo type generalized left fractional derivative with non singular kernel of $\operatorname{order} \rho$, as

$$
D_{a *}^{\rho, \mathscr{F}} f(x):=\underset{\left(\gamma_{j}\right)\left(\omega_{j}\right)}{C A} D_{a *}^{\rho, \lambda, \mathscr{F}} f(x):=\frac{A(\rho)}{1-\rho} \odot \int_{a}^{x} E_{(\rho), \lambda}^{\left(\gamma_{j}\right)}\left[\frac{-\omega_{1} \rho}{1-\rho}(x-t)^{\rho}, \ldots, \frac{-\omega_{m} \rho}{1-\rho}(x-t)^{\rho}\right] \odot f^{\prime}(t) d t, x \in[a, b],
$$

where $A(\rho)$ is a normalizing constant.

Let now $f \in C_{\mathscr{F}}^{n+1}([a, b]), n \in \mathbb{Z}_{+}$. We define the fuzzy Caputo type generalized left fractional derivative with non 
singular kernel of order $n+\rho$, as

$$
D_{a *}^{n+\rho, \mathscr{F}} f(x):=\underset{\left(\gamma_{j}\right)\left(\omega_{j}\right)}{C A} D_{a *}^{n+\rho, \lambda, \mathscr{F}} f(x):=\frac{A(\rho)}{1-\rho} \odot \int_{a}^{x} E_{(\rho), \lambda}^{\left(\gamma_{j}\right)}\left[\frac{-\omega_{1} \rho}{1-\rho}(x-t)^{\rho}, \ldots, \frac{-\omega_{m} \rho}{1-\rho}(x-t)^{\rho}\right] \odot f^{(n+1)}(t) d t, x \in[a, b] .
$$

Similarly, we define the fuzzy Caputo type generalized right fractional derivative with non singular kernel of order $\rho$, as

$$
D_{b-}^{\rho, \mathscr{F}} f(x):=\underset{\left(\gamma_{j}\right)\left(\omega_{j}\right)}{C A} D_{b-}^{\rho, \lambda, \mathscr{F}} f(x):=\frac{-A(\rho)}{1-\rho} \odot \int_{x}^{b} E_{(\rho), \lambda}^{\left(\gamma_{j}\right)}\left[\frac{-\omega_{1} \rho}{1-\rho}(t-x)^{\rho}, \ldots, \frac{-\omega_{m} \rho}{1-\rho}(t-x)^{\rho}\right] \odot f^{\prime}(t) d t, x \in[a, b] .
$$

And, for $f \in C_{\mathscr{F}}^{n+1}([a, b]), n \in \mathbb{Z}_{+}$, we define

$$
D_{b-}^{n+\rho, \mathscr{F}} f(x):={ }_{\left(\gamma_{j}\right)\left(\omega_{j}\right)}^{C A} D_{b-}^{n+\rho, \lambda, \mathscr{F}} f(x):=(-1)^{n+1} \frac{A(\rho)}{1-\rho} \odot \int_{x}^{b} E_{(\rho), \lambda}^{\left(\gamma_{j}\right)}\left[\frac{-\omega_{1} \rho}{1-\rho}(t-x)^{\rho}, \ldots, \frac{-\omega_{m} \rho}{1-\rho}(t-x)^{\rho}\right] \odot f^{(n+1)}(t) d t,
$$

$x \in[a, b]$. The above fuzzy fractional derivatives generalize the fuzzy Atangana-Baleanu type fractional derivatives, see next.

We mention the following non-singular kernel fuzzy fractional derivatives.

Definition 17. Let $f \in C_{\mathscr{F}}^{1}([a, b]), a<b, \alpha \in(0,1)$, the fuzzy Atangana-Baleanu type left fractional derivative is given by

$$
\left({ }^{A B C} D_{a+}^{\alpha, \mathscr{F}} f\right)(x)=\frac{B(\alpha)}{1-\alpha} \odot \int_{a}^{x} E_{\alpha}\left(-\alpha \frac{(x-t)^{\alpha}}{1-\alpha}\right) \odot f^{\prime}(t) d t,
$$

$\forall x \in[a, b]$, where $B(\alpha)$ is a normalizing constant and $E_{\alpha}$ is the one paramjeter Mittag-Leffler function:

$$
E_{\alpha}(x)=\sum_{k=0}^{\infty} \frac{x^{k}}{\Gamma(\alpha k+1)}, x \in \mathbb{R}
$$

which is an entire function converging for all $x \in \mathbb{R}$.

The right side fuzzy fractional derivative is defined by

$$
\left({ }^{A B C} D_{b-}^{\alpha, \mathscr{F}} f\right)(x)=-\frac{B(\alpha)}{1-\alpha} \odot \int_{x}^{b} E_{\alpha}\left(-\alpha \frac{(t-x)^{\alpha}}{1-\alpha}\right) \odot f^{\prime}(t) d t
$$

$\forall x \in[a, b]$.

Let now $f \in C_{\mathscr{F}}^{n+1}([a, b]), n \in \mathbb{Z}_{+}$. We define more generally, the fuzzy left side fractional derivative:

$$
\left({ }^{A B C} D_{a+}^{n+\alpha, \mathscr{F}} f\right)(x)=\frac{B(\alpha)}{1-\alpha} \odot \int_{a}^{x} E_{\alpha}\left(-\alpha \frac{(x-t)^{\alpha}}{1-\alpha}\right) \odot f^{(n+1)}(t) d t,
$$

$\forall x \in[a, b]$, and the fuzzy right side fractional derivative:

$$
\left({ }^{A B C} D_{b-}^{n+\alpha, \mathscr{F}} f\right)(x)=(-1)^{n+1} \frac{B(\alpha)}{1-\alpha} \odot \int_{x}^{b} E_{\alpha}\left(-\alpha \frac{(t-x)^{\alpha}}{1-\alpha}\right) \odot f^{(n+1)}(t) d t,
$$

$\forall x \in[a, b]$. Fuzzy fractional derivatives (74), (76), (77), (78) generalize the following fuzzy Caputo-Fabrizio type nonsingular kernel fractional derivatives. 
Definition 18. Let $f \in C_{\mathscr{F}}^{1}([a, b]), \alpha \in(0,1)$, the fuzzy Caputo-Fabrizio type fractional derivatives have as follows:

$$
{ }^{C F} D_{a+}^{\alpha, \mathscr{F}} f(x)=\frac{1}{1-\alpha} \odot \int_{a}^{x} \exp \left(-\frac{\alpha}{1-\alpha}(x-t)\right) \odot f^{\prime}(t) d t
$$

and

$$
{ }^{C F} D_{b-}^{\alpha, \mathscr{F}} f(x)=-\frac{1}{1-\alpha} \odot \int_{x}^{b} \exp \left(-\frac{\alpha}{1-\alpha}(t-x)\right) \odot f^{\prime}(t) d t,
$$

$\forall x \in[a, b]$. When $f \in C_{\mathscr{F}}^{n+1}([a, b]), n \in \mathbb{Z}_{+}$, we have

$$
{ }^{C F} D_{a+}^{n+\alpha, \mathscr{F}} f(x)=\frac{1}{1-\alpha} \odot \int_{a}^{x} \exp \left(-\frac{\alpha}{1-\alpha}(x-t)\right) \odot f^{(n+1)}(t) d t
$$

and

$$
{ }^{C F} D_{b-}^{n+\alpha, \mathscr{F}} f(x)=\frac{(-1)^{n+1}}{1-\alpha} \odot \int_{x}^{b} \exp \left(-\frac{\alpha}{1-\alpha}(t-x)\right) \odot f^{(n+1)}(t) d t
$$

$\forall x \in[a, b]$.

Finally we give,

Definition 19. Let $f \in C_{\mathscr{F}}^{1}([a, b]), \alpha \in(0,1)$, the fuzzy Atangana-Gomez type fractional derivatives have as follows:

$$
{ }^{A G} D_{a+}^{\alpha, \mathscr{F}} f(x)=\frac{A G(\alpha)}{1-\alpha} \odot \int_{a}^{x} \exp \left(-\frac{\alpha}{1-\alpha}(x-t)^{2}\right) \odot f^{\prime}(t) d t
$$

and

$$
{ }^{A G} D_{b-}^{\alpha, \mathscr{F}} f(x)=-\frac{A G(\alpha)}{1-\alpha} \odot \int_{x}^{b} \exp \left(-\frac{\alpha}{1-\alpha}(x-t)^{2}\right) \odot f^{\prime}(t) d t
$$

$\forall x \in[a, b]$. When $f \in C_{\mathscr{F}}^{n+1}([a, b]), n \in \mathbb{Z}_{+}$, we have

$$
{ }^{A G} D_{a+}^{n+\alpha, \mathscr{F}} f(x)=\frac{A G(\alpha)}{1-\alpha} \odot \int_{a}^{x} \exp \left(-\frac{\alpha}{1-\alpha}(x-t)^{2}\right) \odot f^{(n+1)}(t) d t
$$

and

$$
{ }^{A G} D_{b-}^{n+\alpha, \mathscr{F}} f(x)=\frac{(-1)^{n+1} A G(\alpha)}{1-\alpha} \odot \int_{x}^{b} \exp \left(-\frac{\alpha}{1-\alpha}(x-t)^{2}\right) \odot f^{(n+1)}(t) d t,
$$

$\forall x \in[a, b]$, where $A G(\alpha)$ is a normalization constant.

We make,

Remark. In this work we presented a number of specific new fuzzy fractional integrals and derivatives mostly of nonsingular kernels, see Definitions 15-19. All these are special cases of the fuzzy fractional integrals (27), (28), respectively, we introduced in Definition 13, and we studied in Remark 3 and Theorems 7-11. Therefore all these special cases satisfy all the established general properties of (27), (28), respectively. That is our specific fuzzy fractional entities satisfy (34), (38), they are all fuzzy continuous and satisfy the fuzzy fractional inequalities (43), (50)-(52), respectively.

To keep our presentation short as a sample we mention only the following: The Opial inequalities follow:

Corollary 1. Let $p, q>1: \frac{1}{p}+\frac{1}{q}=1$. All as in Definition 15. Then

$$
\int_{a}^{x} D\left(\left(e_{\rho, \mu, \omega, a+}^{\gamma, \mathscr{F}} f\right)(w), \widetilde{0}\right) D(f(w), \widetilde{0}) d w \leq 2^{-\frac{1}{q}}\left(\int_{a}^{x}\left(\int_{a}^{w}\left[(w-t)^{\mu-1} E_{\rho, \mu}^{\gamma}\left[\omega(w-t)^{\rho}\right]\right]^{p} d t\right) d w\right)^{\frac{1}{p}}
$$




$$
\left(\int_{a}^{x}(D(f(w), \widetilde{0}))^{q} d w\right)^{\frac{2}{q}}, \forall x \in[a, b]
$$

and

(2)

$$
\begin{gathered}
\int_{x}^{b} D\left(\left(e_{\rho, \mu, \omega, b-}^{\gamma, \mathscr{F}} f\right)(w), \widetilde{0}\right) D(f(w), \widetilde{0}) d w \leq 2^{-\frac{1}{q}}\left(\int_{x}^{b}\left(\int_{w}^{b}\left[(t-w)^{\mu-1} E_{\rho, \mu}^{\gamma}\left[\omega(t-w)^{\rho}\right]\right]^{p} d t\right) d w\right)^{\frac{1}{p}} \\
\left(\int_{x}^{b}(D(f(w), \widetilde{0}))^{q} d w\right)^{\frac{2}{q}}, \forall x \in[a, b] .
\end{gathered}
$$

Proof. Apply (43) and (50), respectively.

The Hardy inequalities follow:

Corollary 2. Let $p, q>1: \frac{1}{p}+\frac{1}{q}=1$. All as in Definition 17. Then

(i)

$$
\left\|D\left({ }^{A B C} D_{a+}^{n+\alpha, \mathscr{F}} f(\cdot), \widetilde{0}\right)\right\|_{q,[a, b]} \leq \frac{|B(\alpha)|}{1-\alpha}\left(\int_{a}^{b}\left(\int_{a}^{x}\left(E_{\alpha}\left(-\alpha \frac{(x-t)^{\alpha}}{1-\alpha}\right)\right)^{p} d t\right)^{\frac{q}{p}} d x\right)^{\frac{1}{q}}\left\|D\left(f^{(n+1)}(\cdot), \widetilde{0}\right)\right\|_{q,[a, b]},
$$

and

(ii)

$$
\left\|D\left({ }^{A B C} D_{b-}^{n+\alpha, \mathscr{F}} f(\cdot), \widetilde{0}\right)\right\|_{q,[a, b]} \leq \frac{|B(\alpha)|}{1-\alpha}\left(\int_{a}^{b}\left(\int_{x}^{b}\left(E_{\alpha}\left(-\alpha \frac{(t-x)^{\alpha}}{1-\alpha}\right)\right)^{p} d t\right)^{\frac{q}{p}} d x\right)^{\frac{1}{q}}\left\|D\left(f^{(n+1)}(\cdot), \widetilde{0}\right)\right\|_{q,[a, b]}
$$

Proof. By Theorem 11.

\section{References}

[1] G.A. Anastassiou, Rate of convergence of Fuzzy neural network operators, univariate case, The Journal of Fuzzy Mathematics, 10, No. 3 (2002), 755-780.

[2] G.A. Anastassiou, Higher order Fuzzy Korovkin Theory via inequalities, Communications in Applied Analysis, 10(2006), No. 2, 359-392.

[3] G.A. Anastassiou, Fuzzy Korovkin Theorems and Inequalities, Journal of Fuzzy Mathematics, 15(2007), No. 1, $169-205$.

[4] G.A. Anastassiou, Fractional Differentiation Inequalities, Research Monograph, Springer, New York, 2009.

[5] G.A. Anastassiou, Fuzzy Mathematics: Approximation Theory, Springer, Heidelberg, New York, 2010.

[6] G.A. Anastassiou, Intelligent Mathematics: Computational Analysis, Springer, Heidelberg, New York, 2011.

[7] G. Anastassiou, Advances on Fractional Inequalities, Springer, Heidelberg, New York, 2011.

[8] G.A. Anastassiou, Intelligent Comparisons: Analytic Inequalities, Springer, Heidelberg, New York, 2016.

[9] G.A. Anastassiou, Foundations of Generalized Prabhakar-Hilfer fractional Calculus with Applications, submitted, 2021.

[10] A. Atangana, D. Baleanu, New fractional derivatives with nonlocal and non-singular kernel: theory and application to heat transfer model, Therm. Sci., 20 (2) (2016), 763-769.

[11] A. Atangana and J.F. Gomez-Aguilar, Numerical approximation of Riemann-Liouville definition of fractional derivative: From Riemann-Liouville to Atangana-Baleanu, Numer. Methods. Partial Differ. Equ., vol. 34, no. 5, pp. 1502-1523, 2018.

[12] M. Caputo and M. Fabrizio, A new Definition of Fractional Derivative without Singular Kernel, Progr. Fract. Differ. Appl., vol. 1, no. 2, 73-85, 2015.

[13] S. Gal, Approximation Theory in Fuzzy Setting, Chapter 13 in Handbook of Analytic-Computational Methods in Applied Mathematics, pp. 617-666, edited by G. Anastassiou, Chapman \& Hall/CRC, 2000, Boca Raton, New York. 
[14] A. Giusti et al, A practical Guide to Prabhakar Fractional Calculus, Fractional Calculus \& Applied Analysis, Vol. 23 (1) (2020), 9-54.

[15] Goetschel, R., Jr., and Voxman, W., Elementary fuzzy calculus, Fuzzy Sets and Systems, 18 (1986), 31-43.

[16] R. Gorenflo, A. Kilbas, F. Mainardi, S. Rogosin, Mittag-Leffler functions, Related Topics and Applications, Springer, Heidelberg, New York, 2014.

[17] O. Kaleva, Fuzzy differential equations, Fuzzy Sets and Systems, 24(1987), 301-317.

[18] Y.K. Kim, B.M. Ghil, Integrals of fuzzy-number-valued functions, Fuzzy Sets and Systems, 86(1997), 213-222.

[19] J. Losada, J.J,. Nieto, Properties of a New Fractional Derivative without Singular Kernel, Progr. Fract. Differ. Appl. 1 (2) (2015), 87-92.

[20] R.K. Saxena, S.L. Kalla, Ravi Saxena, Multivariate analogue of generalized Mittag-Leffler function, Integral Transforms and special Functions, 22 (7) (2011), 533-548.

[21] H.M. Srivastava, and M.C. Daoust, Certain generalized Newmann expansion associated with Kompe' de Feriet function, Nederl. Akad. Wetensch. proc. Ser. A 72 (Indag. Math.) 31 (1969), pp. 449-457.

[22] H.M. Srivastava and M.C. Daoust, A note on the convergence of Kompe' de Feriet's double hypergeometric series, Math. Nachr. 53 (1972), pp. 151-159.

[23] H.M. Srivastava and P.W. Karlsson, Multiple Gaussian Hypergeometric Series, Ellis Horwood Limited, New York, 1985.

[24] C. Wu, Z. Gong, On Henstock integral of fuzzy-number-valued functions (I), Fuzzy Sets and Systems, 120, No. 3, (2001), $523-532$.

[25] C. Wu, M. Ma, On embedding problem of fuzzy numer spaces: Part 1, Fuzzy Sets and Systems, 44 (1991), 33-38. 